IX.

Aus der I. med. Klinik und dem physiologischen Institut der Universität Wien.

\title{
Über den Einfluss der Kastration auf den Blutbefund weiblicher Tiere.
}

\author{
Von \\ Dr. Robert Breuer und Dr. Rudolf Freih. v. Seiller.
}

Die Lehre von der internen Sekretion hat sich mit ziemlich gutem Erfolg in den Dienst der ätiologisehen Forschung gestellt und ist für die Pathogenese mancher Krankheiten mit Glüek verwertet worden. Es handelt sich hier um ihrem Wesen nach so gut wie unbekannte, doch fur den normalen Lebensprozel notwendige Funktionen drüsiger Organe, deren Aussehaltung oder deren gestörter Ablauf eine mehr oder minder empfindliche Schädigung des Organismus herbeifuhrt. Für einige dieser Drüsen (Schilddrüse und Nebenniere) steht eine chemische Beeinflussung bestimmter Stoffwechselvorgänge außer allem Zweifel.

Auch die weiblichen Keimdrtisen sind in neuerer Zeit mit der Entstehung einer Reihe von Konstitutionsanomalien in einen ursächlichen Zusammenhang gebracht worden. Sie sollen aufer ihrer wohlbekannten Funktion der Orulation noch eine zweite Aufgabe der inneren Sekretion besitzen und durch eine hypothetische, von ihnen produzierte Substanz die Stoffwechselvorgänge im Körper beeinflussen. Der Wegfall oder der gestörte Ablauf dieser inneren Sekretion der Ovarien soll einer Reibe von Konstitutionskrankheiten: Osteomalacie, Fettleibigkeit, Chlorose zugrunde liegen.

Hier sei nur von der letztgenannten Krankheit, der Chlorose, die Rede.

Es ist bekannt, daß das:Bestreben, die Chlorose mit den Genitalorganen in einen pathogenetischen Zusammenhang zu bringen, sehr alt ist und daß solehe Beziehungen schon zu einer Zeit konstruiert wurden, wo der Gedanke an eine innere Sekretion der Organe, hier 
der Eierstöcke, also an einen chemischen Einfulb dieser Organe auf den tubrigen Körper, noch in weiter Ferne lag. Das ausschließliche Vorkommen der Chlorose beim weiblichen Geschlecht, ihr überwiegend häufiges zeitliches Zusammentreffen mit dem Pubertätsalter macht es ja auch begreiflich, daß sie schon von ibren ältesten Beobachtern mit der sexuellen Sphäre des Weibes in Verbindung gebracht wurde; dafür sprechen schon die älteren Bezeichnungen der Krankheit, wie Morbus virgineus, Pallor virgineus, Ikterus amantium, Febris amatoria usw.

Auch in neuerer Zeit fehlen die Bestrebungen nicht, dem in die Augen springenden Zusammenhang der echten Bleichsucht mit Vorgängen in der Geschlechtssphäre gerecht zu werden und wir besitzen (von den neuesten Hypothesen abgesehen, die auf eine innere Absonderung der Ovarien rekurrieren) eine ganze Reihe von "genitalen Theorien" der Chlorose.

Bestimmtere Anschaunngen über die Relationen zwischen Chlorose und weiblichem Genitalapparat begegnen wir znerst bei Virchow (2). In seiner bekannten Abhandlung macht er auf die bei Chlorose ungemein häufig anzutreffende und nicht selten von Entwicklungsstörungen des Genitalapparates begleitete Hypoplasie des Gefäßsystems, insbesondere des Herzens und der großen Arterienstämme aufmerksam 1): "Abgesehen von denjenigen Fällen von Chlorose, in welchen der Sexualapparat keine gröberen Abweichungen darbietet, findet man Zustände von $\mathrm{mangel}$ hafter Ausbildungdes zentralen Teiles des GefäBapparates bei gleichzeitiger Mangelhaftigkeit des Geschlechtsapparates; aber man findet auch umgekehrt eine große, ja exzessive Entwicklung des Geschlechtsapparates bei derselben Mangelhaftigkeit des Gefäßsystems." Wie Virchow meint, kämen hier hauptsächlich die Orarien in Betracht, weniger der Uterus. Er fährt dann fort: „Wenngleich ich nicht in Abrede stellen will, daß eine gewisse Beziehung sich festhalten läßt, so muß doch noch geprüft werden, wo eigentlich das primum movens liegt; ob die Mangelhaftigkeit des Uterus auf die Ovarien influenziere oder umgekehrt, und weiterhin, ob die Einwirkungen, welche der Geschlechtsapparat auf die übrigen Körperteile ausübt, das $\mathrm{MaB}$ der Ausbildung des Blutes und des Gefäßapparates bestimmen, oder ob vielmehr primäre Mängel des Blutes und des Gefäßapparates auf die Ausbildung des Sexualapparates wirken. Letzteres hat gewiß manches für sicl." Virchow läßt es hiermit unentschieden, ob die Entwicklungsstörungen der Genitalorgane als etwas

1) Auf ein vereintes Vorkommen von Bildungsmängeln des Gefäß- und Sexualapparats - namentlich beim weiblichen Geschlechte - hat zuerst Rokitan sky (1) hingewiesen; wie es in seinem Handbuche der pathologischen Anatomie heißt, trifft ,regelwidrige Kleinheit des Herzens und des Aortensystems" . . . ,überhaupt nicht selten, vorzüglich aber bei diesem" (i. e. dem weiblichen Geschlechte) ,mit allgemein zurückgebliebener Entwickelung und namentlich mit zurückgebliebener Entwickelung der Sexualorgane zusammen". 
Primäres oder als etwas Sekundäres, durch die Hypoplasie des vaskulären Apparates bedingt, anzusehen seï.

E. Fränkel (3) berichtet uber einen Fall von Chlorose mit sehr rudimentärer Entwicklung des Genitalapparates ,jedoch ohne mangelhafte Bildung des Herzens und der großen Gefäße" und spricht sich im Gegensatz zu Virchow dahin aus, daß die Sexualaplasie "das Maß der Ausbildung des Blutes bestimmen und somit an und für sich das Primum movens der Chlorose sein kann".

Neuerdings ist Stieda (4) dieser Frage näher getreten. Er suchte an dem Material der gynäkologischen Poliklinik zu Freiburg festzustellen, ob Zustände, die wir als Hemmungsbildungen oder Entwicklungsstörungen ("Degenerationszeichen") bezeichnen miissen, bei Chlorotischen insbesondere am Becken, an den Brüsten und an den Genitalien hänfiger vorkommen als bei anderen. Die an einer, wenn auch kleinen Zahl von Fällen gewonnenen Ergebnisse sind sehr bemerkenswert: Bei 23 Chlorotischen waren in 73,9 Proz., bei 233 Nichtehlorotischen in 20 bis 27,5 Proz. der Fälle vereinzelte oder mehrere Entwicklungsstörungen anzutreffen; insbesondere waren die 0 varien bei den Chlorotischen 12 (!) Mal, bei den Nichtchlorotischen nur zweimal mangelhaft entwickelt.

Nach der Ansicht Stiedas stehen die ,genuine Chlorose, welche sich nicht auf äußere Schädlichkeiten oder primäre Leiden zurückführen läßt" und die bierbei häufig. vorkommenden Hemmungsbildungen an den Genitalien nicht in einem Verhältnis gegenseitiger Abhängigkeit; sie sind vielmehr einander koordiniert und als Ausdruck einer gemeinsamen Schädlichkeit, die den Organismus vielleicht schon in der allerfrühesten Zeit seiner Entwicklung oder im Keime getroffen hat, aufzufassen.

Nach einer anderen Richtung weisen die Erwägungen von Beneke (5) und Murri (6).

Beide legen das Hauptgewicht anf reflektorische nervöse Impulse, die - von den zur Zeit der Pubertät sich üppig entfaltenden weiblichen Sexualorganen ausgehend - die blutbildenden Organe beeinflussen. Sie treffen nach. B e neke (5) zunächst die Organe des Digestionstraktes, insbesondere die Leber; die Folge hiervon ist eine mangelhafte Assimilation des Eisens resp. ein abnormer Verlust desselben. Beneke legt hierbei viel Gewicht auf den in der Leber erfolgenden Abbau des Hämoglobinmoleküls und auf den damit zusammenhängenden Untergang roter Blutkörnchen, der die Hämoglobinverarmung des Blutes, die Chlorose, bedingen soll. Nach Murri (6) kommt es dureh eine von "den Genitalorganen ausgehende reflektorische Alteration der vasomotorischen Zentren zu einer ungleichmäßigen Blutverteilung im Sinne einer peripheren Ischämie und einer zentralen Hyperämie und infolge dieser Zirkulationsstörung zu einem vermebrten Zerfall der roten Blutzellen, die ja bekanntlich auf eine veränderte Blutzusammensetzung sehr leicht reagieren. Auch die Kälte wirkt hämolytisch, daher die große Zahl der Winterchlorosen.

Im letzten Jahrzehnt, unter dem Einflusse der Lehre von der internen Sekretion und der mächtig sich entwickelnden Serumforschung traten eine Reihe von Autoren für chemische Wechsel- 
beziehungen zwischen den weiblichen Geschlechtsorganen und dem hämatopoetischen Apparat ein, deren gestörter Ablauf einen schädlichen Einfluß auf die Blutbereitung ausübe und das Symptomenbild der Chlorose hervorrufe.

Daß den Ovarien tatsächlich außer ihrer Funktion der Eibildung ein Einfluß auf den übrigen Körper zukommt, steht aus verschiedenen Gründen, namentlich klinischer Natur (man vergleiche die Erscheinungen der künstlichen und natürlichen Menopause) wohl schon seit langem fest. Und daß die Orarien befähigt sind, auf chemischem Wege, d. h. durch innere Sekretion, Wirkungen auszuitben, unterliegt jetzt nach den Stoffwechselversuchen (CaratuloTarulli [7], Senator [8], P. F. Richter [9], Thumim [10], Dalché-Lépinois [11], Neumann-Vas [13], schließlich Loewy und Richter [14]) keinem Zweifel. Die Transplantationsversuche (Knauer [15] u. a.) sprechen in demselben Sinne. Dafür jedoch, daß diese innere Sekretion in einer Beziehung zur Blutbildung stehe, liegen, wie aus der folgenden Zusammenstellung hervorgeht, außer vielfachen, zum Teil höchst bypothetischen theoretischen Erörterungen bis jetzt nur eine Anzahl von therapeutisehen Versuchen vor, die sich auf den günstigen Einfluß der Fütterung von Ovarialsubstanz auf den Verlauf von Chlorosen beziehen. Und auch diese Versuche sind in ihren Resultaten wenig übereinstimmend und im ganzen wenig beweiskräftig.

Charrin (16) hat als erster auf die Möglichkeit eines ursächlichen Zusammenhanges zwischen der Chlorose und einer inneren Sekretion der Ovarien hingewiesen: Er führt die Chlorose auf eine Autointoxikation seitens der Genitalorgane zurück. Vor dem Eintritt der Menses wächst die Toxizität des Serums, durch die Menses werden die Toxine ausgeschieden; bleiben jene ans, so kommt es durch die im Körper zurïckgehaltenen Giftstoffe zur chlorotischen Erkrankung des Organismus. Der Autor verweist auf die Diarrhöe und anderer Störungen der Säuglinge bei Amenorhöe der Ammen.

Eine Bekräftigung dieser Ansicht wäre in den leider viel zu wenig ausgedehnten Untersuchungen von Leclere und Levet (17), zweier Schüler Charrins, zu finden, nach denen die Toxizität des Blutserums bei Chlorose größer sei als bei normalen Individuen. Gegenüber $15 \mathrm{ccm}$ Serum eines gesunden Mädchens genügten in 4 Fällen $11 \mathrm{ccm}$, in einem Falle $8,5 \mathrm{ccm}$ chlorotischen Serums, um $1 \mathrm{~kg}$ Kaninchen zu töten.

Einmal durch Charrin angeregt, hat die Idee, daß der Chlorose eine gestörte sekretorische Funktion der Ovarien zugrunde liegen dürfte, namentlich in Frankreich Anklang gefunden; es haben sich in den folgenden Jahren eine Anzahl französischer und italieniseher Autoren mit dieser Frage beschäftigt und eine mehr oder minder umgrenzte Beantwortung derselben zu geben versucht. 
Die ersten, die (unter Anlehnung an die Charrinsehe Theorie) die interne Sekretion der Ovarien ausdrücklich in Betracht ziehen, sind Spillmann and Etienne (18). Nach ihnen haben die Ovarien neben ihrer Hauptfunktion - der Ovulation - die Aufgabe, toxische Substanzen im Wege der menstruellen Blutungen auszuscheiden und durch innere Sekretion die allgemeine Ernährung des Körpers zu beeinflussen. Die Chlorose ist als eine durch Menstruationsstörungen (Amenorrhöe) bedingte Autointoxikation aufzufassen. Führt man dem Organismus Produkte der inneren Sekretion der Ovarien zu, so ließe sich vielleicht dadurch die Intoxikation beheben und die Heilung der Ovarien und somit der Chlorose herbeiführen. Unter den mit Ovarialsubstanz be. handelten Chlorosen besserten sich drei Fälle ziemlich rasch. Blut. befunde werden niebt mitgeteilt.

Fedeli (19) hält die Chlorose für eine durch mangelhafte innere Sekretion der Ovarien bedingte Neurose. Soweit sich ans der nicht sehr klaren und etwas gezwungenen Deduktion entnehmen läßt, wären für eine normale Blatbildung bestimmte, die blutbildenden Organe beeinflussende Impulse des Nervensystems notwendig, die aber nur durch die Produkte der inneren Sekretion der Ovarien ausgelöst werden; andererseits ist dann auch das Nervensystem imstande, die Ovarien zu neuer Tätigkeit anzuregen, so daß hier die Reize in einem Zirkel ablaufen. Bei gestörter innerer Sekretion kommt es zum Ausbruch der Chlorose mit all ihren anämischen und nervösen Symptomen; durch Verabreichung von Ovarin läßt sich die innere Sekretion der Ovarien und somit die Krankheit günstig beeinflussen.

Muret (20) hat die bekannten Beschwerden bei physiologischer und artifizieller Menopause durch innere und subkutane Verabreichung von Ovarialpastillen und Ovarialextrakt mit sehr gutem Erfolg bekämpft und auch in 4 Fällen von Chlorose eine Besserung des Befindens und des Blutbefundes erzielt. Er hält das Bestehen einer internen Sekretion der Ovarien für sehr wahrscheinlich; sie dürfte für die normale Entwicklung und das regelrechte Funktionieren des weiblichen Organismus nützlich, wenn nicht notwendig sein. Fehlen oder atrophieren die Ovarien, so bedarf der Organismus einer längeren oder kürzeren Zeit, um wieder sein Gleichgewicht zu finden, das vielleicht durch eine andere Drüse mit interner Sekretion wiederhergestellt wird.

Blondel (21) bekämpft die Theorie Charrins. Viele amenorrhoische Frauen and Mädchen, ja solche, die niemals menstruiert wurden, werden nicht chlorotisch; andererseits sind viele Chlorotische ganz normal, ja sehr stark menstruiert. Die Amenorrhöe ist nicht die Ursache, sondern die Folge der Chlorose. Diese sei als eine durch Stoffwechselstörungen bedingte Intoxikation aufufassen; die Toxine entstehen nur während des Wachstums des Körpers, später nicht mehr; sie werden durch die. sekretorische Tätigkeit der Thymus, und wenn diese obliteriert ist, durch jene der Ovarien unschädlich gemacht, Obliteriert die Thymus, ehe die Ovarien ihre Tätigkeit entfalten, so bricht in diesem Interregnum die Chlorose aus.

Auf Grund seiner (12) Harnanalysen hält Salmon (22) die Chlorose für eine Antointoxikation, bedingt durch einen trägen Stoffwechsel im 
Sinne einer verminderten Oxydation. Infolge mangelhafter Verbrennung der Säuren werden die Körpersäfte und der Harn hyperazid: die Chlorose entwickelt sich immer nur in einem übersäuerten Organismus. Die Ovarien sezernieren (nach Poehl und Etienne u. Demange) einen Stoff von stark oxydierender Beschaffenheit; bei mangelhafter Funktion, i. e. gestörter innerer Sekretion, kommen die erwähnten Stoffwechselanomalien zur Geltung und erzeugen die Chlorose.

Unter dem Vorbehalt, nur eine Hypothese auszusprechen, führt v. Noorden (23) die Chlorose auf den Ausfall oder die Abschwächung einer "internen Sekretion "zurück: "Im weiblichen Organismus, der alle 4 Wochen erhebliche Mengen Blut durch die Sexualorgane abgibt, sind normalerweise Vorkehrungen getroffen, den Blutverlust schnell und vollständig wieder zu ersetzen. Die Anregungen zur Blutneubildung gehen von den Geschlechtsorganen (Ovarien?) selbst aus und zwar nicht auf reflektorischem Wege, sondern durch Erzeugung chemischer Stoffe, die in das Blut und an die Stätte der Blutbildung gelangen und die Eigenschaft haben, die blutbildenden Organe anzuregen. Die blutbildenden Organe sind zwar nicht ausschließlich auf diese von den Genitalien herkommenden Erregungen angewiesen, aber ihr Ausfall gefährdet doch immer den normalen Fortgang der Blutneubildung, insbesondere bei jugendlichen Individuen. Beim Wegfall oder bei Absehwächung der Erregung kommt es zur Chlorose, d. h. zu einer Insuffizienz der Blutneubildung, die durch spezifische Vorgänge im Genitalapparat veranlaBt wird. Wenn von Haus aus die blutbildenden Organe mangelhaft veranlagt oder durch andere ungünstige Einflüsse geschwächt sind, so wird die Krankheit natürlich um so leichter entstehen. Über die Natur der chemischen Wechselbeziehungen zwischen Sexualapparat und blutbildenden Organen lälot sich einstweilen gar nichts anssagen ..... Jetzt, wo wir über die Bedeutung der sogenannten "internen Sekretionen" einiges wissen und die große Rolle, die sie im Organismus spielen, ahnen, darf die Berechtigung einer Hypothese nicht abgelehnt werden, die die Chlorose auf den Ausfall oder die Abschwächung einer „internen Sekretion" zurïckführt."

Etienne u. Demange (24) dagegen weisen - gleich Salmon in ihrer Theorie der Chlorose der internen Sekretion eine ganz bestimmte Rolle zu, ohne allerdings für ihre Ansicht irgendeinen Beweis zu erbringen. Die Ovarien (vergleiche oben Spillmann und Etienne [18]) haben die Ovulation, die Abscheidung von Toxinen, die sich im weiblichen Körper in exzessivem Maße bilden und die "innere Sekretion" zu besorgen; das Produlkt der letzteren ist "a a point de vue clinique" ein lösliches Ferment mit stark oxydierenden Eigenschaften (analog dem Spermin Poeh1). Bleibt die innere Sekretion wälrend der Entwicklungsepoche des weiblichen Organismus aus, so entsteht ein Autointoxikationszustand unter dem klinischen Bilde der Chlorose. Vor der Pubertät wird die antitoxische Tätigkeit der Ovarien wabrscheinlich von der Thymus besorgt. - Bei 17 Chlorosen wurden durch Ovarienfütterung gute therapentische Erfolge erzielt; nähere Angaben über den Decursus morbi fehlen.

Touvenaint (25) und de Gottal (26) berichten über eine 
güustige Wirkung des Ovarins bei Amenorrhöe, Dysmenorrhöe, physiologischer und artifizieller Menopause und Chlorose. Ihre Mitteilungen beziehen sich hauptsächlich auf das subjektive Befinden der Patientinnen und auf die Besserung der Allgemeinsymptome. Blutbefunde fehlen.

Nach Versuchen mit Eierstockssubstanz in verschiedenen Formen bält Jakobs (28) den Ovarienwein (hergestellt aus Ovarien von Kühen, $20 \mathrm{~g}$ [ $=0,20$ Ovariensubstanz] pro die) für das tauglichste Präparat zur Behandlung der Störungen der natürliehen und postoperativen Menopause, ferner gewisser Fälle von Chlorose und von nervösen Störungen, die mit der Menstruation in Beziehung stehen. Chlorotische und Dysmenorrhoische fühlen eine rasche Besserung ihres Leidens. Keine Blutbefunde.

Dagegen haben Schaumann und v. Willebrand (27) bei zwei von drei mit Ovarialtabletten durch 12 und 33 Tage behandelten Fällen von Chlorose nur ein mäßiges Ansteigen der Erythrozyten (um 500000 und 800000) und des Hämoglobins (um 4 Proz. und 17 Proz. Fleischl) beobachtet.

Man sieht, daß auch unter den Vertretern der neweren ,genitalen“ Auffassung der Chlorose die Ansichten weit auseinander gehen. Nur eines haben alle diese Erklärungsversuche miteinander gemein: sie sind durchwegs vollkommen bypothetisch und entbehren eigentlieh jedes Beweises.

Es ist zu verwundern, daß noch nie der Versuch gemacht worden ist, etwa auf experimentellem Wege einen solchen Beweis für einen Einfluß der Ovarien auf die Blutbeschaffenheit zu erbringen 1). Vor allem liegt es ja selır nahe, nachzusehen, ob die Zusammensetzung des Blutes etwa durch die Entfernung der Ovarien verändert wird.

Wir haben uns nun vor jetzt 3 Jabren die Aufgabe gestellt, in exakter und einwurfsfreier Weise zu ermitteln, ob und welchen Einfluß die Entfernung der Ovarien auf die Blutbeschaffenheit weiblicher Tiere ausübt. Es war natürlich absolut nicht vorauszusehen, ob diese Versuche ein positives Resultat ergeben würden und nach welcher Richtung hin sich der Einfluh der Kastration kenntlich machen würde; es mag sogar ausdrücklich bemerkt werden, daß wir mit der Meinung an die Arbeit gingen, daß der Erfolg ein ganz anderer sein werde, als er sich tatsäehlich ergeben hat.

Auf keinen Fall aber ließ und läßt sich erwarten, daß die Entfernung der Ovarien im Tierversuch etwa einen bestimmten ans der menschlichen Pathologie bekannten Krankheitszustand zur Folge haben werde. Das war denn auch durchaus nicht der Fall,

1) Auf' einige in unzulänglicher Weise durchgeführte Versuche dieser Art, die inzwischen von italienischen Autoren veröffentlicht worden sind, kommen wir am Schlusse dieser Arbeit zurück. 
und wir möchten eigens betonen, daß wir durch die Entfernung der Ovarien bei unseren Versuchstieren anscheinend uberhaupt keine Krankheit erzeugt haben, daß das Allgemeinbefinden, der Appetit, das Körpergewicht usw. der Tiere keine Veränderungen darbot.

Auch das Blutbild zeigte, wie dargetan werden wird, nicht etwa die Anomalien, die der menschlichen Chlorose eigentümlich sind. Aber es ergaben sich doch so merkwürdige und vor allem konstante Veränderungen der Blutbeschaffenheit nach der Kastration, daß ihre Mitteilung geboten erscheint. Und, haben wir auch bei unseren Tieren nach diesem Eingriff durchaus keine Chlorosen entstehen sehen, so ist es doch nicht ausgeschlossen, daß durch diese Versuche ein Stück der (wahrsoheinlich viel verwickelteren) Pathogenese der Chloranämie beim menschlichen Weibe aufgehellt werden wird.

Unsere Experimente wurden im Laboratorium der I. med. Klinik begonnen und im physiologisehen Institute zu Wien weitergeführt, dessen Vorstand, Herr Hofrat Prof. Exn er, uns alle Hilfsmittel, deren wir bedurften, in liebenswürdigster Weise zur Verfügung stellte, wir sind ihm, sowie seinen Assistenten, vor allem Herrn Prof. A. Kreidl, zu besonderem Danke verpflichtet.

Bevor wir die Ergebnisse unserer Versuche mitteilen, erscheint es nötig, über das verwendete Tiermaterial, über die Methodik der Kastration und der Blutuntersuchungen und zwar über letztere aus bestimmten Grïnden in detaillierterer Weise, zu berichten.

Als Versuchstiere benutzten wir ausschließlich Hündinnen, und zwar, nachdem sich im Beginn der Arbeit bei einigen älteren Tieren absolut kein konstanter Einfluß der Kastration ergeben hatte, junge weibliche Tiere im Alter von ca. 6-10 Monaten, also etwa um die Zeit der Pubertät.

Die Tiere wurden in der Weise ausgewählt, daß nur solche, bei denen sieh die Blutentnahme korrekt und sicher bewerkstelligen ließ (siehe unten), zu den Versuchen herangezogen wurden. - Jedes Tier wurde nach seiner Aufnahme in das Institut einer gewöhnlich mehrwöchentlichen Beobachtungszeit unterzogen, während der (in der Regel in Intervallen von einigen Tagen) das Körpergewicht bestimmt und Blutuntersuchungen in der später zu schildernden Weise vorgenommen wurden. Die Tiere hatten dabei, was uns bei allen derartigen Versuchen, besonders an jungen Tieren, sebr wichtig scheint, die Möglichkeit, sich viel auch außerhalb des Stalles frei bewegen zu können. Auch nach der Operation wurde, sobald die Wunde geheilt war, den Hunden Gelegenheit zum 
Aufenthalt im Freien gegeben. Nur wenn das Tier während der Beobachtungszeit vol' der Operation vollkommen gesund geblieben war, und wenn sein Körpergewicht sowie Hämoglobingehalt und Blutkörperchenzahl sich während dieser Zeit entweder konstant erhalten oder zugenommen hatten, wurde es weiter verwendet und der Kastration, resp. einer Kontrolloperation unterzogen.

Die Operationen wurden in Morphium-Chlorformnarkose, selbstverständlich unter strenger Asepsis und möglichst rasch vollzogen. Der Blutverlust ist bei einiger Übung ein ganz minimaler, auch war jede Nachblutung ausgeschlossen. Die Kastration sowohl, wie die Totalexstirpation des Uterus, und die Exstirpation eines Stückes Netz wird von den Tieren sehr gut vertragen. Störungen des Allgemeinbefindens waren immer auf pathologische Vorgänge (Eiterungen, Katarrbe der Respirationswege usw.) zurückzuführen, und solche Tiere wurden von der weiteren Verwendung ausgeschlossen. Bei glatter prima intentio und ohne accidentelle Krankheiten waren die Ticre schon am Tage nach der Operation ganz munter und bei gutem Appetit. Die operierten Tiere wurden unter möglichst gleiche Bedingungen gesetzt, wie vor der Operation (Qualität der Nahrung, Maß der Bewegung usw.) und sorgfältig beobachtet; nur solohe Exemplare, bei denen der Heilungsverlauf und der Allgemeinzustand nichts zu wünschen tubrig ließen und bei denen das Gewicht auch nach der Operation konstant blieb oder zunahm, wurden zu weiteren Blutuntersuchungen herangezogen.

Solche ganz tadellose Hunde zu erhalten ist nun durchaus nicht so leicht. Bei den meisten physiologiseh-chirurgischen Versuchen fält eine mäBige Wundeiterung kaum ins Gewicht, man übersieht sie daher leicht und bezeichnet den Operationserfolg als einwandsfrei. Richtet man aber sein Augenmerk auf derartige Dinge, so begegnet man ihnen aucb bei größter Sorgfalt recht häufig; da es sich nun nicht von der Hand weisen läßt, daß selbst eine Störung des Wundverlaufs, die das Allgemeinbefinden weiter nicht beeinflußt, eine ungünstige Wirkung auf die Blutbeschaffenheit austiben könnten, so mußte, obwohl von unserer Seite alles geschah, um solche Störungen hintanzuhalten, denuoch leider so manche Reihe von Untersuehungsergebnissen schließlich als nicht ganz einwandfrei ausgeschieden und die aufgewendete Mühe und Zeit von Wochen verloren gegeben werden.

Nur die vorübergehende Sekretion einiger Tropfen Eiter aus einem einzelnen oberflächlicben Stichkanal in der Haut, so- 
wie eine vorübergehende, etwas stärkere, seröse Absonderung der Wunde haben wir in einigen Fällen nicht zum Anlaß genommen, das betreffende Tier zu verwerfen.

Wir haben übrigens später bei den Kontrollversuchen die Erfahrung gemacht, daß selbst mäßige eitrige Sekretion von mehreren Stichkanälen in der Hautwunde, wie sie sich zufällig bei einem der Kontrolltiere einstellte, auf die Blutzusammensetzung gar keinen Einfluß babe. Doch empfahl es sioh immerhin, in den Kastrationsversuchen möglichst rigoros vorzugehen, und wir haben deshalb auch in der nachfolgenden Zusammenstellung nur diejenigen kastrierten Tiere angefithrt, bei denen der Wundverlauf ein absolut tadelloser war und eine Reihe von Hunden nicht beriicksichtigt, bei denen zwar derselbe Effekt eintrat wie bei den tadellosen Tieren, bei denen aber Wundeiterungen, Hautkrankheiten nsw. uns das Resultat nicht einwandsfrei erseheinen ließen.

Über die systematischen Blutuntersuchungen, die an den Tieren, wie bemerkt, stets mehrmals vor der Operation und dann nach dem Eingriff in kurzen Zwischenräumen vorgenommen wurden, haben wir folgendes zu bemerken:

Wir hatten anfangs versucht, Blut aus größeren Gefäßen (Venen oder Arterien) zur Untersuchung zu verwenden. Da sich das Aufsuchen von tieferliegenden Gefäßen, vor allem wenn es in kurzen $\mathrm{Z}$ wisehenräumen oft wiederholt werden muß, nicht wohl bewerkstelligen läßt, ohne daß die Blutentnahme oder ihre Folgen selbst schon zu einer Schädigung und damit zur Trübung der Resultate fïhren können, entnahmen wir im Anfang unserer Arbeit das Blut aus Venen des Ohres. Es stellte sich jedoch bald heraus, daß diese Methode absolut unverwendbar war. Wir erhielten bei Untersuchungen an demselben gesunden Tier so inkonstante Werte, die Blutkölperchen- und Hämoglobinzahlen differierten an aufeinander folgenden Tagen so bedeutend, daß wir diesen Weg aufgeben mußten. Nach vielen fruchtlosen Versuchen hat sich als das einzig korrekte Verfahren die Untersuchung von Kapillarblut erwiesen, das dureh einen Einstich mit einem nicht zu kleinen, ziemlich breiten und sehr scharfen Lanzenmesser in die Pfote gewonnen wird. Doch eignet sich zu dieser Methode der Blutentnahme (und damit zu den Untersnchungen überhaupt) durchaus nicht jedes Tier.

Man entnimmt das Blut am besten aus den Ballen einer Vorderpfote; dabei ist genau darauf zu achten, daß das Blut sofort nach dem Einstich in einem großen Tropfen hervorquillt. Dies geschieht nun durehaus nicht immer; manche Hunde haben eine sehr fett- 
reiche, in ihren oberen Sclichten gefäßarme Kutis, oder die Pfoten sind schlaff und mager; in beiden Fällen sickert das Blut nur langsam aus der Tiefe des Einstichs hervor; die Bestimmungen sind dann ganz unzuverlässig, sie fallen - meist - zu hoch aus; offenbar weil den Blutkörperchen Zeit gelassen wird zu sedmentieren, sie gelangen dann in größerer Zahl in die Mischpipette; oder zu niedrig, wenn man das Blut aus den oberen Schichten des Tropfens entnimmt. Solche Hunde sind natürlich ganz untanglich; besonders im Anfang mußten wir viel Lehrgeld zahlen und manche Tiere früher oder später aus unseren Versuchsreihen aussehalten. Später gewinnt man einige Übung in der Beurteilung der Pfoten und kann sich dann unangenehme Enttäusehungen und manchen Ärger ersparen. Ubrigens geht auch bei ganz verläßlichen Pfoten durchaus nicht jedesmal alles nach Wunsch; in solchen Fällen wurde natürlich auch von einer Blutbestimmung aus dem betreffenden Einstich Abstand genommen.

Der erste Tropfen nach dem Einstich wurde mit einem reinen Tuche abgewischt und erst das hieranf ohne jeden Druck gutfliebende Blut aufgesaugt. Die Pfote mußte natürlich rein und ganz trocken sein; eine Reinigung mit Alkohol und Äther wurde grundsätzlich nicht angewendet.

Zur Bestimmung des Hämoglobingehaltes bedienten wir uns des Hämometers von Fleischl-Miescher, das sich uns in diesen Tierversuchen (wie auch in zabllosen Untersuchungen am Menschen während der letzten Jahre) als ein ganz ausgezeichneter und verläßlicher Apparat erwiesen hat. Bei Einhaltung der Vorsehriften, die namentlieh Veillon (34) empfollen hat, haben wir den festen Eindruck gewonnen, daß - korrekte Blutentnahme vorausgesetzt - die Fehler der Bestimmungen mit unserem Instrument uiber wenige Promille des absoluten Hämoglobingehaltes bestimmt nicht hinausgingen.

Alle unsere Hämoglobinzahlen bedeuten im folgenden absolute Hämoglobinwerte (Gewichtsprozente).

Die Zahl der roten Blutkörperchen wurde aus dem Mittel von 10 großjen (160 kleinen) Quadraten der T ho ma-Z e i schen Zählkammer bestimmt. Es sei bier bemerkt, daß nur große Übung, peinlichste Akkuratesse bei raschem Arbeiten und das genaue Einhalten bestimmter Vorschriften (Größe des Tropfens, Auflegen des Deckglases, Verteilung der $\mathrm{N}$ e w to n schen Ringe, Vermeidung jeder uiberflüssigen Bewegung des Präparats usw.) verläßliche Resultate gewährleisten. 
In vor kurzem publizierten Untersuchungen kommt Bräning (33) zu dem Schlub, daß alle Blutkörperchenzählungen, die mit der üblichen Methodik in der Thoma-Zeißschen Kammer vorgenommen werden, mit außerordentlich großen Fehlern behaftet seien, weil von dem Moment des Aufbringens des Tropfens auf den Kammertisch bis zum Auflegen des Deckglases die Blutkörperchen sedimentieren und dann in dem fertigen Präparat ungleichmäßig verteilt sind: in den zentralen Partien dichter, in den peripheren in größeren Zwischenräumen liegen. Brünings hat eine Kammer konstruiert, in der sich eine vollkommen gleichmäßige Verteilung der Körperchen erzielen läßt.

Brünings Untersuchungen sind sicher außerordentlich interessant und wertroll. Doch können wir nicht glauben, daß dadurch die Resultate aller Zählungen in der alten Kammer, wenn sie nur sorgfältig und mit gewissen Kantelen vorgenommen worden sind, entwertet werden. Jeder, der viel Blutkörperchen gezählt hat, weiß, daß die Zählmethode mit der Thomaschen Kammer relativ wenig verläßlich und schon bei geringem Mangel an Sorgfalt mit großen Fehlern behaftet ist. Auch wir sind der Ansicht, daß ein großer Teil der in der Literatur niedergelegten Zählungsresultate nur als Annäherungswerte aufzufassen sind. Wir haben deshalb alle Vorsichtsmaßregeln stets genau eingehalten und insbesondere Gewicht darauf gelegt, daß den Körperchen zum Sedimentieren möglichst wenig Zeit gelassen werde. Nach dem Erscheinen der Brüningssehen Arbeit haben wir die Zeit, die bei unserer Art zu arbeiten zwischen dem Aufbringen des Tropfens und seiner Verteilung durch das Auflegen des Deckglases verstrich, bestimmt und gefunden, daß sie nicht mehr als höchstens 5 Sekunden betrug. Dazn muß allerdings (was bei uns immer der Fall war) alles, vor allem das geputzte Deckglas vor dem Auftragen des Tropfens schon bereit liegen. Auf gleichmäßige Verteilung der Körperchen im Präparat haben wir stets sorgsam geachtet und wir glauben, daß man die Verteilung bei einiger Übung und bei Durchmusterung mit $\mathrm{schwacher}$ Vergrößerung doch recht wohl beurteilen kann. Jedes Zählpräparat, das uns nicht gleichmäßig schien, haben wir verworfen.

Wir sehen einen Beweis dafut, dalo unsere Zählresultate trotz der Ausführungen Brünings wohl brauchbar sind, darin, daß Unregelmäßigkeiten (zickzackförmiges Ansteigen und Absinken der Werte, wie es bei größeren Fehlern notwendig vorkommen mußte) in den Blutkörperchenwerten bei unseren Versuchstieren vor der Kastration, also während des Normalzustandes der Tiere, fast gar nicht zu beobachten waren, vor allem aber darin, daß sich fast durchwegs ein weitgehender Parallelismus der Körperchenkurve mit der Kurve der Hämoglobinwerte zeigt, welch letztere wir allerdings, wie oben bemerkt, für recht verläßlich halten.

Wir gehen nunmehr zur Besprechung unserer Versuche tiber. Diese erstrecken sich auf 2 Reihen von Beobachtungen:

1. Kastrierte Tiere,

2. Kontrollversuche, in welchen statt der Kastration eine andere abdominale Operation vorgenommen wurde. 
Was die Kastrationsversuche anlangt, so stehen uns 13 nach jeder Richtung einwandsfreie Tiere zur Verfügung. Dies scheint auf den ersten Blick nicht sehr viel zu sein. Bedenkt man jedoch, daß zu den ca. 200 Hämoglobinbestimmungen and ebensovielen Blutkörperchenzählungen an diesen Tieren noch ein paar bundert Bestimmungen kommen, deren Resultate nicht verwertet werden konnten, weil sich die betreffenden Tiere später als unbrauchbar erwiesen, weil sie interkurrent erkrankten, oder weil der Heilungsverlauf der Operationswunde kein absolut tadelloser war, so wird man es begreiflich finden, daß wir nach ea. 3 jähriger Arbeit uns mit den erwähnten 13 Versuchen zufrieden gaben. Das war um so eher möglich, als diese Versuche, wie aus dem folgenden hervorgehen wird, alle in demselben Sinne sprachen und weil - was wir eigens hervorheben möchten - sich auch nie die Andeutung eines entgegengesetzten Resultates ergab.

Das Verhalten der Blutwerte bei den kastrierten Tieren wird am besten aus den folgenden Beispielen ersichtlich.

$$
\text { Hündị Nr. I. }
$$

Datum

11. September

Rote

Blutkörperchen

7800000

7950000

Absoluter

14.

Kastration am 14. September.

17. September
19. =
$24 .=$
$27 .=$
30. "
3. Oktober
$7 . \quad=$
$10 .=$
$16 .=$

6500000

6600000

5700000

5900000

5950000

7000000

7000000

6850000

7000000

Hämoglobingehalt

$172 / 3$ Proz. $18^{2} / 3=$

Hündin Nr. X.
Datum
Rote
Absoluter
Blutkörperchen
Hămoglobingehalt
30. Mai
7400000
$16^{2} / 3$ Proz.

$161 / 6$ Proz.

$161 / 2=$

$16^{2 / 3}=$

15

$142 / 3=$

$15 \frac{1 / 3}{16}=$

$16^{1 / 6}=$

$171 / 3=$

19

Kastration am 1. Juni

$\begin{array}{rll}\text { 4. Juni } & 7550000 & 16^{2} / 3 \text { Proz. } \\ 6 .= & 6800000 & 171 / 6= \\ 13 .= & 7350000 & 162 / 3= \\ 18 .= & 6850000 & 16= \\ 24 .= & 5550000 & 14= \\ 27 .= & 4950000 & 13=\end{array}$




\begin{tabular}{|c|c|c|c|}
\hline Datum & $\begin{array}{c}\text { Rote } \\
\text { Blutkőrperchen }\end{array}$ & \multicolumn{2}{|c|}{$\begin{array}{l}\text { Absoluter } \\
\text { Hămoglobingehalt }\end{array}$} \\
\hline 2. Juli & 5250000 & 12 & Proz. \\
\hline $9 .=$ & 3800000 & $91 / 3$ & $=$ \\
\hline 16. $=$ & 4950000 & 10 & $=$ \\
\hline 19. $=$ & - & 10 & $=$ \\
\hline 25. $=$ & 4300000 & $9^{2 / 3}$ & $=$ \\
\hline $30 .=$ & 3950000 & $10^{1 / 3}$ & $=$ \\
\hline 3. August & 5200000 & 10 & $=$ \\
\hline 12. $=$ & 5050000 & 13 & $=$ \\
\hline $19 .=$ & 5800000 & $13^{1 / 3}$ & $=$ \\
\hline 3. September & 6100000 & $15^{1 / 2}$ & $=$ \\
\hline
\end{tabular}

Die angeführten beiden Beispiele zeigen, daß das Absinkeu der Blutwerte oft ein recht bedeutendes war.

Als drittes Beispiel führen wir absichtlich dasjenige Tier an, bei dem das Absinken der Blutwerte zwar noch deutlich, aber (besonders bei der Blutkörperchenzahl) viel geringer war, auch nicht unmittelbar nach der Kastration, sondern erst etwas später eintrat (wir kommen auf diesen Punkt weiter unten noch zurück). Es handelte sich um ein etwas älteres Tier.

$$
H \text { ü } n \text { din Nr. XII. }
$$

\begin{tabular}{|c|c|c|c|}
\hline Datum & $\begin{array}{c}\text { Rote } \\
\text { Blutkörperchen }\end{array}$ & \multicolumn{2}{|c|}{$\begin{array}{l}\text { Absoluter } \\
\text { Hämoglobingehalt }\end{array}$} \\
\hline 4. Januar & 6900000 & 18 & Proz. \\
\hline 14. $=$ & 7050000 & 18 & $=$ \\
\hline \multicolumn{4}{|c|}{ Kastration am 14. Januar. } \\
\hline 17. Januar & 7050000 & 18 & Proz. \\
\hline 23. & 6500000 & $18^{1 / 3}$ & 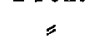 \\
\hline 27. & 6600000 & $16 / / 3$ & $=$ \\
\hline 30. & 7100000 & 17 & $=$ \\
\hline 3. Februar & 7500000 & $17^{1 / 3}$ & $=$ \\
\hline
\end{tabular}

Hier sind (wahrscheinlich des höheren Alters des Versuchstieres wegen) die Verhältnisse, die in den beiden früher mitgeteilten Beispielen und bei einer Anzahl anderer Tier sehr markant zum Ausdruck kamen, gewissermaßen nur angedeutet.

Man sieht aus diesen Beispielen vor allem, dah nach der Kastration Hämoglobingebalt und Zahlder Blutkörperchen beträchtlich oder doch wenigstens deutlich absinken.

Dieses Sinken der Blutwerte trat bei allen kastrierten Tieren ausnahmslos ein.

Wir sehen daher von einer detaillierten Wiedergabe der Versuchsprotokolle hier ab, und stellen die Resultate in Form von $\mathrm{Kurven}$ dar (vergl. Kurve 1-13). 
Über den Einfluß der Kastration auf den Blutbefund weiblicher Tiere. 183

Kurve 1.

Hündin I. Kastration am 14. September.

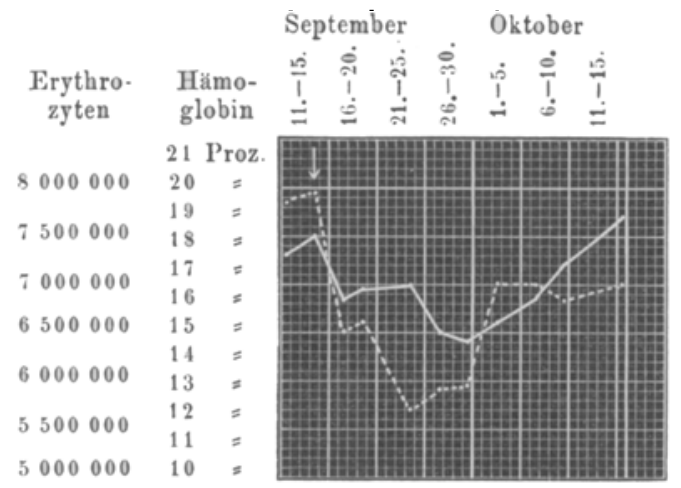

Kurve 2. Hündin II. Kastration am 27. September.

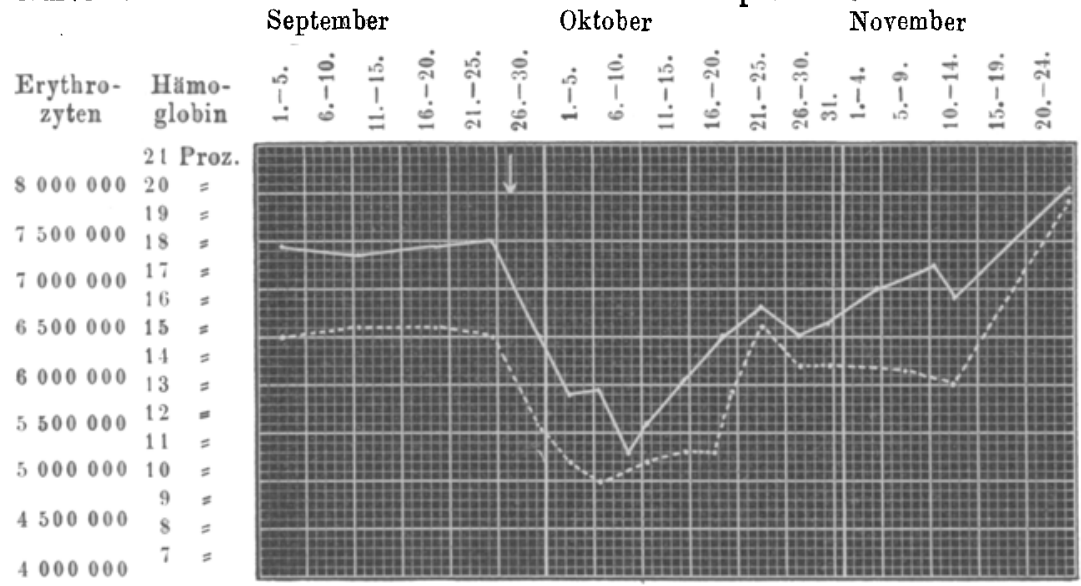

Kurve 3. Hündin III. Kastration am 2, Februar.

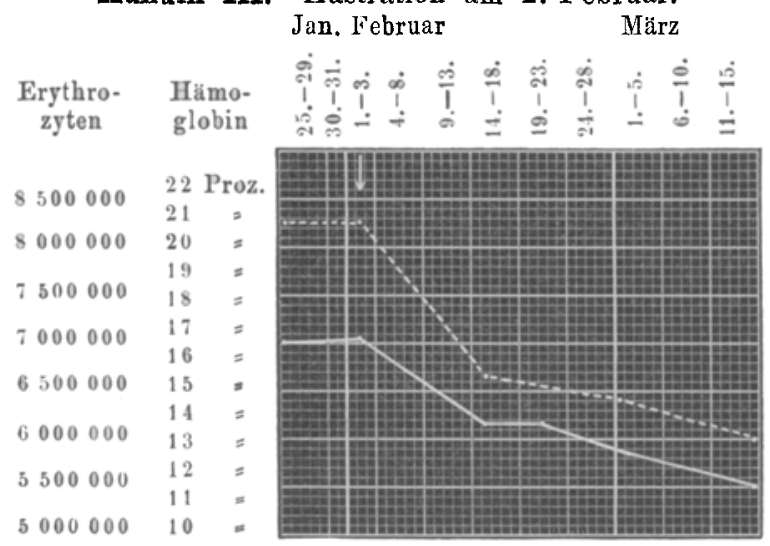




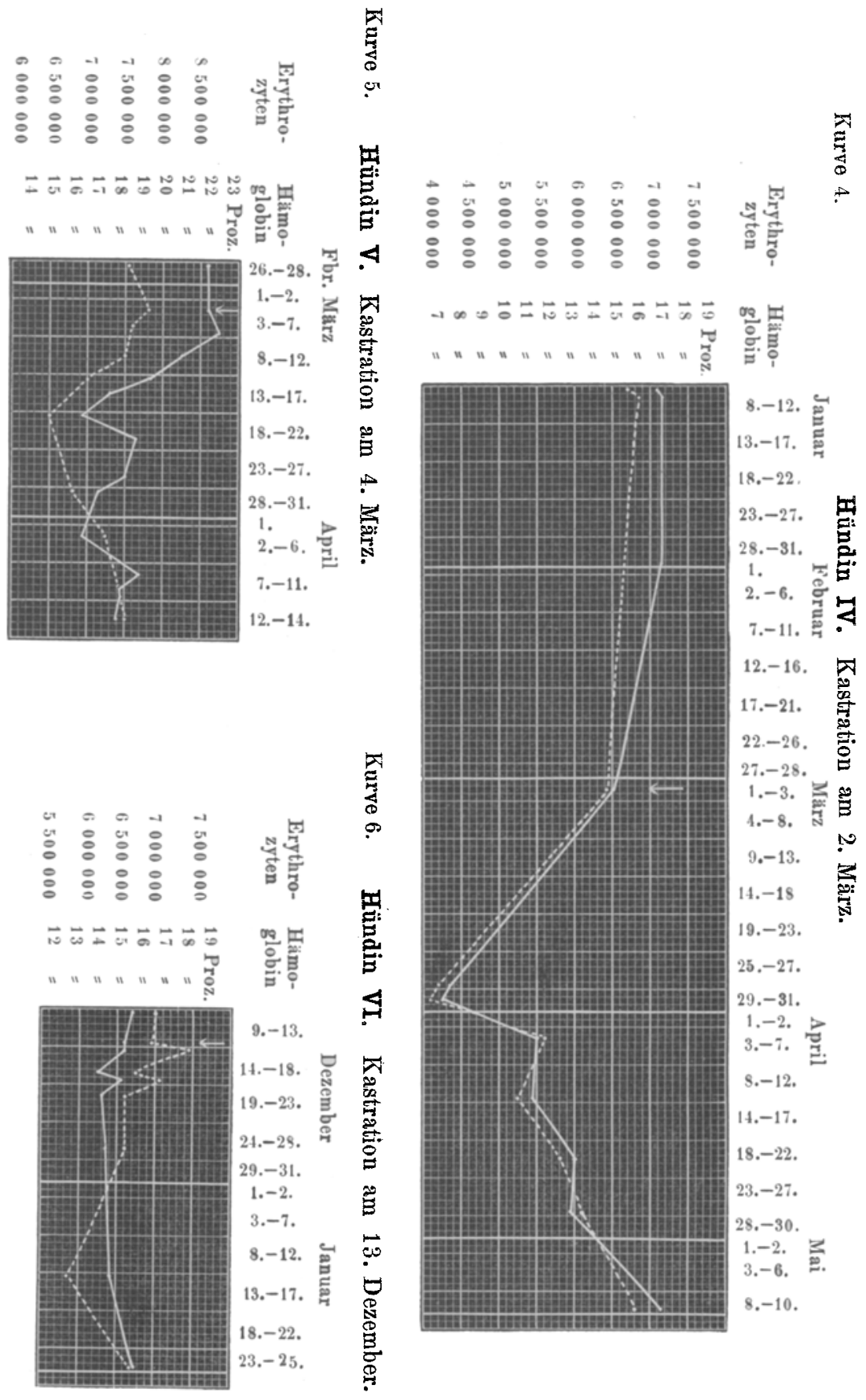


Über den Einfluß der Kastration anf den Blutbefund weiblicher Tiere.

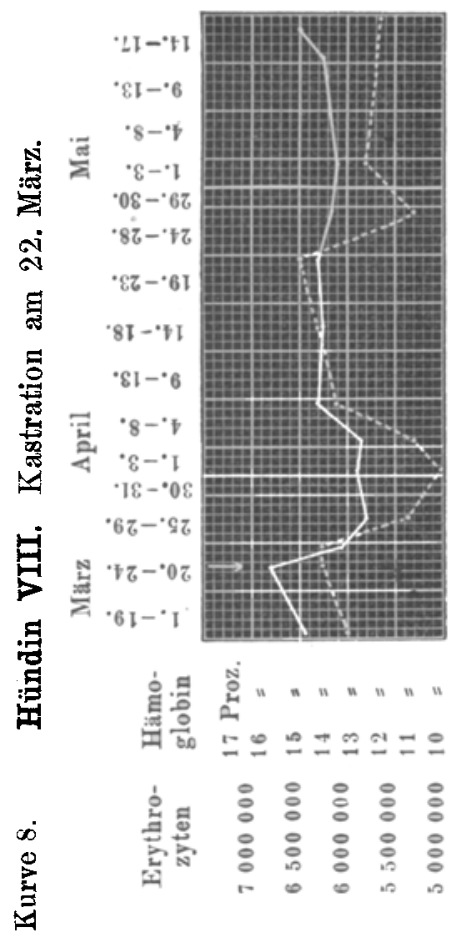

Hündin IX. Kastration am 5. Januar.

\section{Kurve 9.}

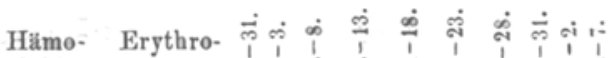
globin zyten

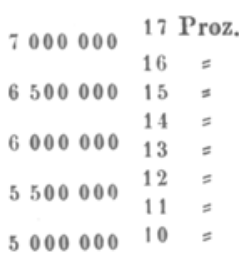
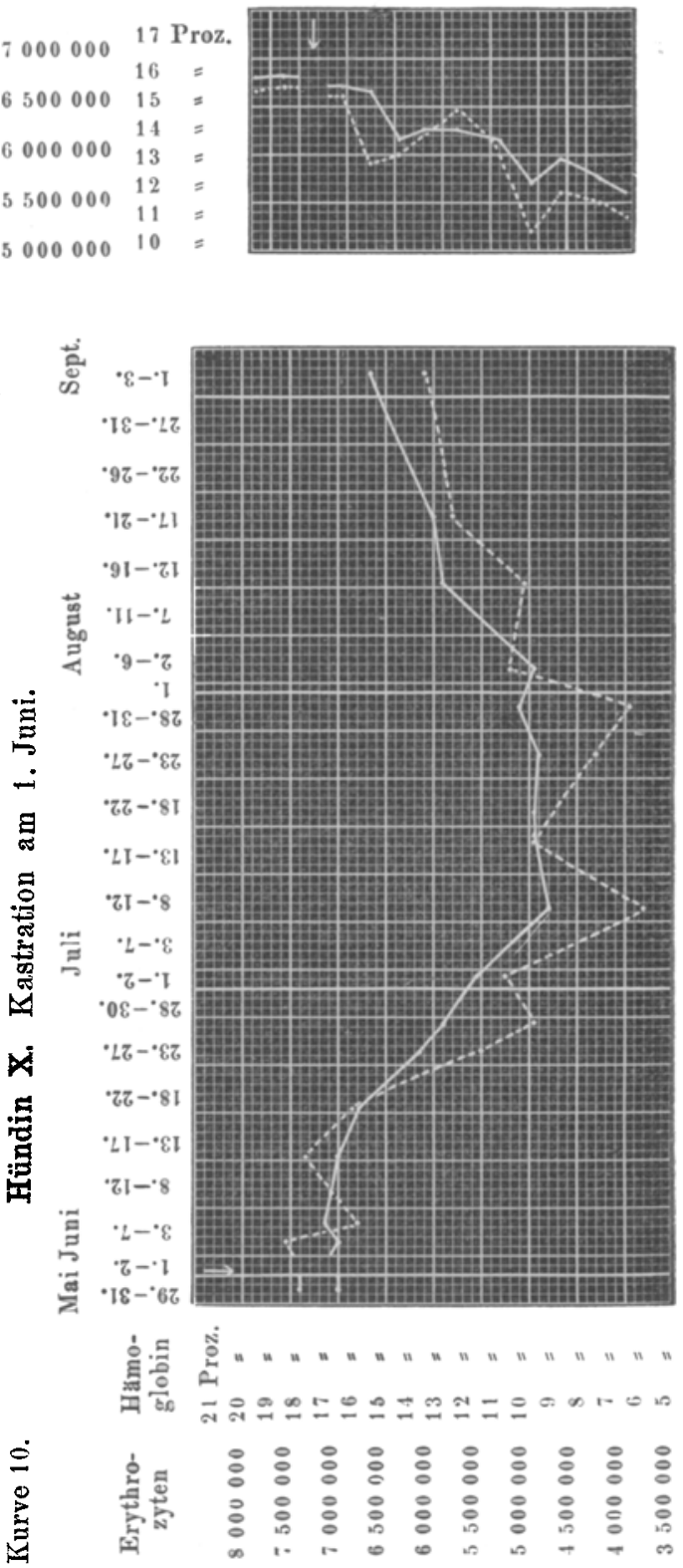


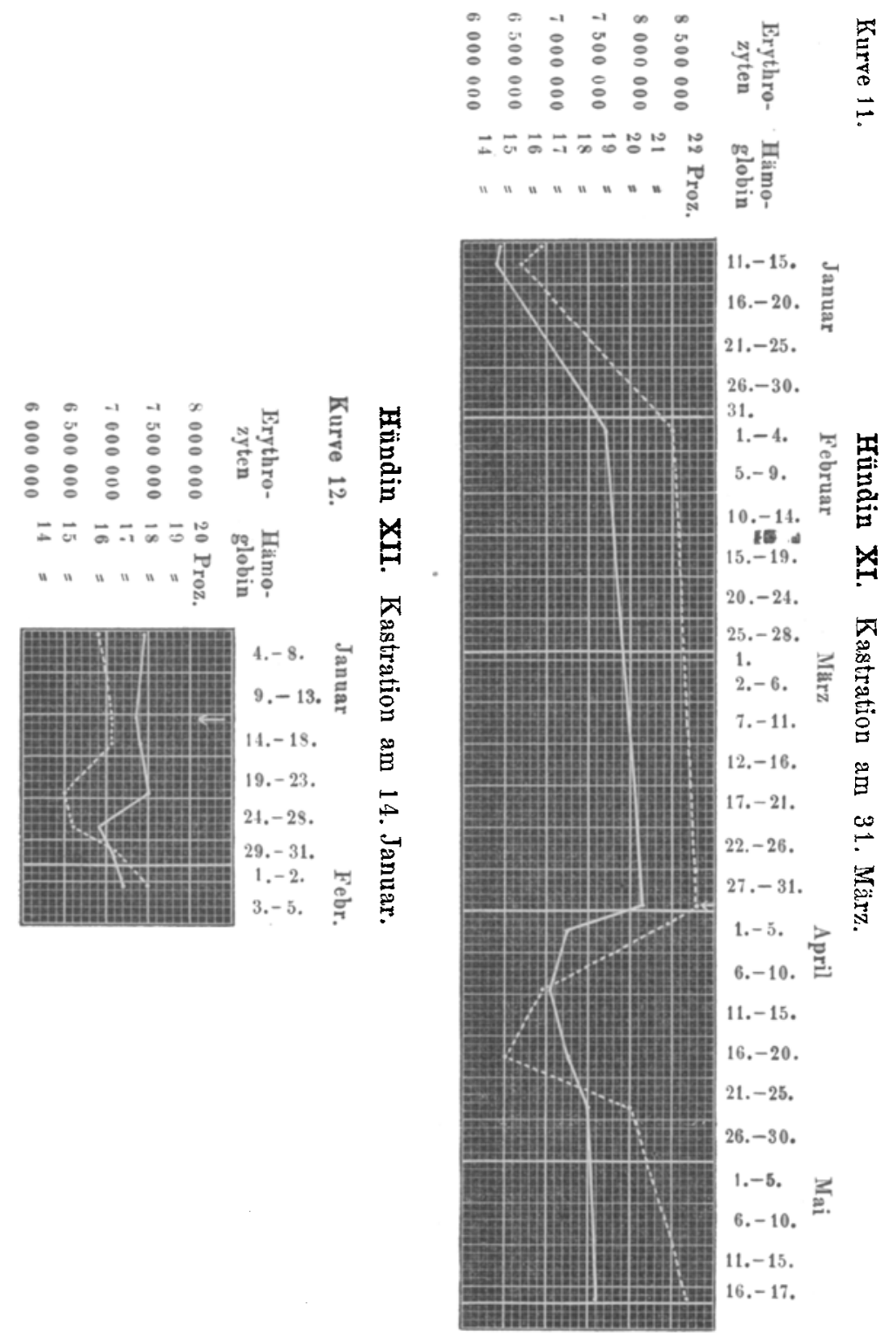

Zum Verständnis dieser Kurven möchten wir folgendes bemerken: Hämoglobingehalt und Blutkörperchenzahl jedes Tieres wurden in dasselbe Liniensystem eingezeichnet. Dabei nahmen wir nach den Erfahruugen bei der Mehrzabl der gesunden Tiere an, daß: 
Über den Kinfluß der Kastration auf den Blutbefund weiblicher Tiere, 187

8000000 Erythrozyten 20 Proz.,

$\begin{array}{ccc}6500000 & =15= \\ 5000000 & 15= \\ 3500000 & 10= \\ \text { Hämoglobin (Gewichtsprozente) } \\ \text { entsprechen. }\end{array}$

bei jedem Tiere zutrifft, sondern daß bei manchen gesunden Individuen der Färbeindex der Erythrozyten ein etwas höherer oder niedriger ist, ergibt sich aus den Anfangsstïcken einiger Kurven, z. B. XIII, XI, V, II).

Von den beiden Linien stellt stets die glatt ausgezogene den Verlauf der Hämoglobinwerte, die punktierte den der Körperchenzahlen dar.

Die Blutwerte in der meist mehrwöchentlichen Beobachtungszeit vor der Operation sind nur in einigen Kurven sämtlich eingezeichnet (z. B. in II, IV, XI, XIII); die anderen Tiere, bei denen eine vorläufige Blntuntersuchung niedrige Werte ergab und der Ernährungszustand ein solcher war, daß man die niedrigen Blutwerte eventuell ihm zur Last legen konnte, wurden wochenlang im Institut gehalten. Bei ihnen sind nur die letzten Werte vor der Operation, die gewissermaßen die Normalwerte für das betreffende Individuum bei gutem Ernäbrungszustande darstellen, in die Kurve aufgenommen.

Abgeschlossen wurden unsere Versuche im allgemeinen dann, wenn nacb dem dureh die Kastration bewirkten Absinken die Blutwerte ungefähr wieder auf ihre frühere Höhe zurückgekehrt waren. Bei einigen Tieren mubte der Versuch aus äußeren Gründen (Überfullung

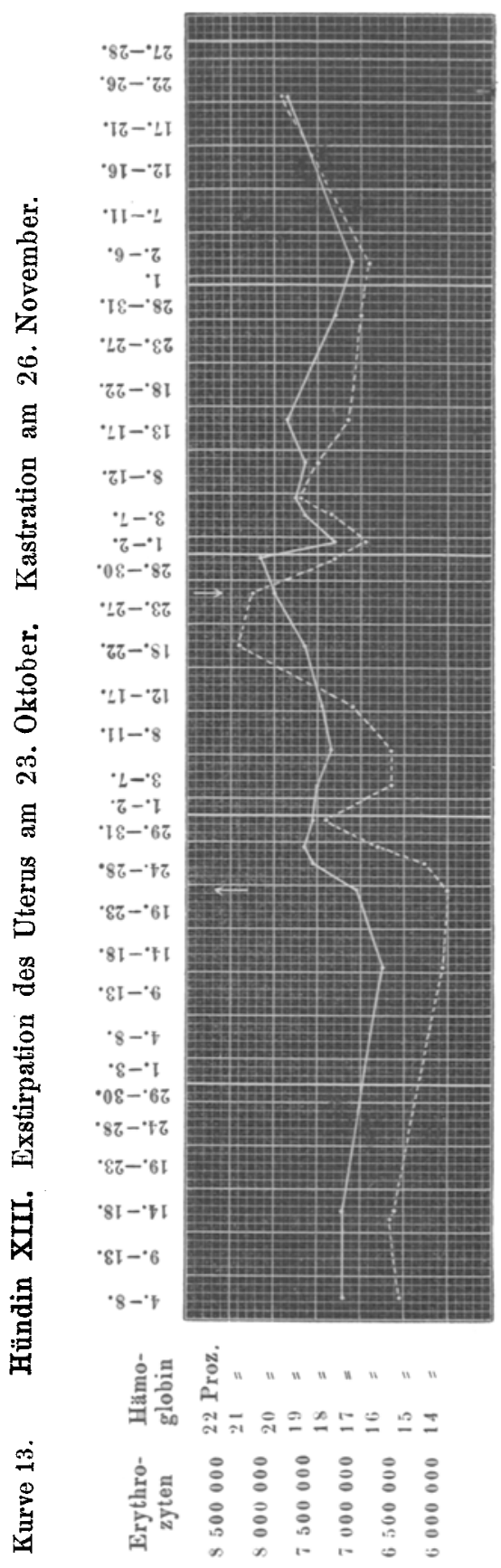


des Stalles mit Versuchstieren) schon etwas früher abgebrochen werden; zweimal (in Fall III und IX) mußten die Blutuntersuchungen schon früher, während die Kurve noch abstieg, eingestellt werden, weil eine schwere Verletzung dureh ein anderes Tier resp. eine plötzliche akut fieberhafte Erkrankung die Blutwerte von diesem Zeitpunkte an nicht mehr beweisend erscheinen ließ. -

Wir gehen nun zur detaillierteren Besprechung und Diskussion der Resultate über:

Der Abfall der Werte des Hämoglobins und der Erythrozyten ging in den einzelnen Fällen ziemlich parallel, d. h. der Färbeindex des Blutes bleibt nach der Kastration ziemlich unverändert (im Gegensatz zur typischen menschlichen Chlorose). Bei den vorübergehenden Divergenzen, die auf einigen Kurven zu sehen sind, macht es den Eindruck, als ob der Blutkörperchenwert als der leichter beeinflußbare um den Hämoglobinwert herumschwanke. Doch ist hier zu bemerken, daß Fehler bei der Körperchenzählung, der vielen Fehlerquellen der Methode wegen, vielleicht auch einem Teil der Unregelmäßigkeiten zugrunde liegen dürften, während die regelmäßig und meist ohne plötzliche Knickungen verlaufende Hämoglobinkurve der tatsächlichen Blutveränderungen wohl genau entspricht.

Der Beginn des Abfalls der Blutwerte fällt meistens in die ersten Tage nach der Kastration - nicht immer (vergl. die Kurven $\mathrm{V}$, IX, X, in denen in den ersten Tagen die Werte gleichbleiben oder sogar ein wenig ansteigen). Es ist natürlich möglich, daß das plötzliche Absinken der Blutwerte, wie es in der Regel rasch nach der Operation auftritt, ein kombinierter Effekt des Verlustes der Eierstöcke und des Traumas der Operation sei. Daß das wesentliche dabei aber das Fehlen der Ovarien ist, und der Eingriff nur vielleicht das plotzliche Einsetzen der Blutveränderungen verschuldet, wird später noch ausführlich bewiesen werden. Außerordentlich wichtig aber ist, daß das Absinken in der Mehrzahl der Fälle noch wochenlange allmählich und gleiehmäßig andauerte, nachdem die eventuelle Schädigung des operativen Eingriffes:längst vor über sein mußte. Wie oben erwähnt wurde, befanden sich die Tiere fast regelmäßig am Tage nach der Kastration so wohl, daß sie umberliefen, fraßen, und trotz des Fastens am Tage der Operation gar keinen Gewiohtsverlust erkennen ließen. Nach einer Woche konnten sie wohl durchwegs als vollkommen gesund gelten und doch dauerte bei aus- 
gezeichnetem Allgemeinbefinden und unter manchmal recht bedeutender Gewichtszunahme das Absinken der Blutwerte nach wochenlange an und der tiefste Stand wurde z. B. vom Tiere Nr. IV erst nach 28, von $\mathrm{Nr}$. X erst nach 40 Tagen erreicht.

Der Anstieg der Werte, nachdem der tiefste Punkt erreicht war, vollzog sich in versehieden langer Zeit, die zwischen 2 und 8 Wochen schwankte; - je tiefer der Abfall, desto länger dauerte es gewöhnlich, bis das Blut auf dem status quo ante angelangt war.

Was dies Maß der Verminderung der Blutwerte nach der Kastration betrifft, so; schwankt es recht beträchtlich, ohne daß wir imstande wären zu sagen, warum das eine Tier unter im übrigen gleichen Verhältnissen um so viel stärker reagiert habe, als das andere. Nur das eine läßt sich sagen, daß im allgemeinen Tiere, die älter waren (etwa gegen ein Jahr), träger und weniger ausgiebig zu reagieren schienen, als solche zwischen $1 / 2$ und $3 / 4$ Jahren. Die Verminderung des absoluten Hämoglobingehaltes beträgt in den wenig prägnanten Fällen (z. B. VI, VII) nicht viel über 1 Proz., die Abnahme der Körperchenzahl nicht über eine Million. Dagegen ist in den Fällen II, IV, X eine Verminderung des absolnten Hämoglobinwertes um 7-10 Proz. (das entspräche etwa einen Abfall des relativen Hämoglobingehaltes bestimmt mit dem "gewöhnlichen Hämometer von Fleischloder Gow ers von 45-70 Proz.) und eine Verminderung der Körpercherzahl um 21/2-31/2 Millionen zu beobachten.

In einigen unserer Fälle (z. B. VIII, V) stellten die Kurven (und zwar auch die Hämoglobinkurve) keine annähernd regelmäßig abund dann wieder ansteigenden Linien dar, wie in den anderen Fällen, sondern sie verlaufen mit starken Schwankungen, Kurve VIII zeigt ein 2 maliges Absinken und Wiederansteigen. Worauf dieser Verlauf zu beziehen sei, wissen wir nicht. Man wird sich vielleicht vorstellen können, daß die Einwirkung, die die Ovarien nach allem auf die Konstanterhaltung des Blutzustandes offenbar besitzen, einige Zeit nach der Kastration vikariierend von anderen Organen übernommen werde, und daß in den fraglichen Fällen dieses vikariierende Eintreten nur unregelmäßig und zunächst nicht dauernd erfolgt sei. Doch das ist vorläufig mehr als hypothetisch und es empfiehlt sich wohl nicht, darauf näher einzugehen.

Die Resultate unserer Untersuchungen lassen sich kurz dahin zusammenfassen, dab nach der Kastration junger Hündinnen ausnahmslos-bei völligem Woblbefinden der Tiere und bei gleichbleibenden oder zu. 
nehmendem Körpergewicht ein Sinken, sowohldes Hämoglobingehaltes des Blutes als der Körperchenzahl ein. trat 1 .

Wir haben unsere Untersuebungen über den Einfluß der Kastration auf das Blutbild von vorneherein auf den Hämoglobingehalt und die Blutkörperchenzahl beschränkt und können deshalb über eventuelle Veränderungen der Leukozytenzahl oder uiber morphologische Veränderungen des Blutbildes, sowie über den Zustand der hämatopoetisehen Organe, z. B. des Knochenmarkes, keine genaueren Angaben machen. So viel läßt sich aber aus der Betrachtung der Zählpräparate wohl behaupten, daß wesentliche Veränderungen in diesen Beziehungen nicht vorgelegen haben dürften. Bei einigen Versuchstieren mit sehr starkem Abfall der Erythrozytenzahlen (z. B. Tier Nr. X), haben wir zurzeit des Tiefstandes und der Regeneration einzelne kernhaltige rote Körperchen rom Typus der Normoblasten gesehen und auch in gefärbten Trockenpräparaten festgestellt.

Das ist weiter nicht verwunderlich, denn es handelt sich z. B. in Fall X um eine Reduktion der Körperchenzahl um mehr als die Hälfte (das entspräche etwa beim Menschen einer Verminderung der Erythrozytenzahl von 4,5 Millionen auf knapp 21/4 Millionen.

War es auch nach dem ganzen Verlauf von vorneherein recht unwahrscheinlich, daß die Veränderungen in der Blutbeschaffenheit nach der Kastration nicht auf Rechnung der Entfernung der Ovarien kämen, sondern als Folge der Laparotomie resp. der Narkose aufzufassen seien, so haben wir es doch für notwendig gehalten, uns in dieser Beziehung durch einige Kontrollversuche sicherzustellen. (Vergl. No. XIII-XVI S. 191.)

In Fall XIV und XV wurde bei jungen weiblichen Tieren, die nach denselben Gesichtspunkten ausgewählt waren, wie jene für die Kastration, die supravaginale Amputation des Uterus per la-

1) Wir bemerken ausdrücklich, daß dieses Sinken der Blutwerte nicht nur in den mitgeteilten 13 Versuchen, sondern auch bei jenen kastrierten Tieren erfolgte, die schließlich verworfen werden mußten, weil sich bei ihnen nicht ausschließen ließ, daß das beobachtete Sinken der Blutwerte vielleicht von dem nicht ganz einwandsfreien Wundverlauf oder von einer interkurrenten Affektion (Husten, Hautkrankheit) herrühre. Aber ausnahmslos bei je d e m kastrierten Tier stellte sich das erwähnte Sinken der Blutwerte ein, $n$ i c h t e in m a l war etwa (wie in den später besprochenen Kontrollversuchen) ein Steigen zu beobachten, und darum können die mitgeteilten Versuche tatsächlich als vollkommen beweisend gelten. 
Üher den Einfluß der Kastration auf den Blutbefund weiblicher Tiere. 191 parotomiam vorgenommen. Die Tuben wurden an ibrem uterinen Ende doppelt ligiert und durchschnitten.

Hündin XIV. Exstirpation des Uterus am 25, Mai .

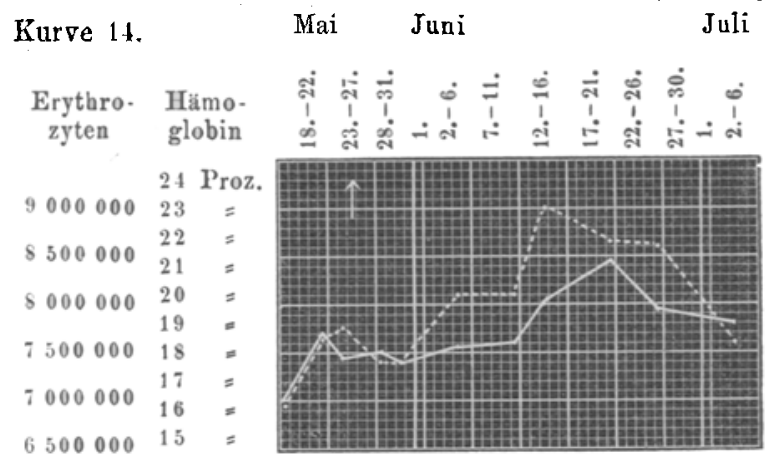

Hündin XV. Exstirpation des Uterus am 22. Mai.

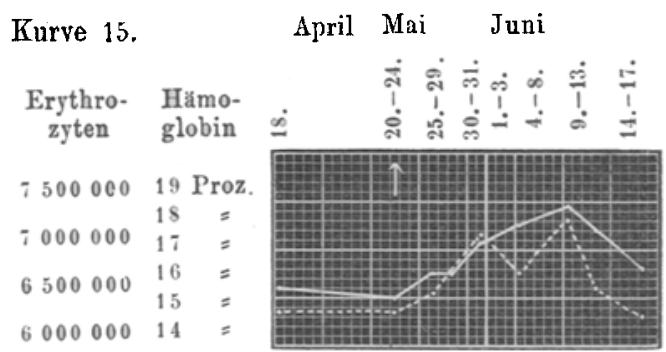

Hündin XVI. Resektion eines Stückes Netz am 12. Mai.

Kurve 16.

Mai Juni

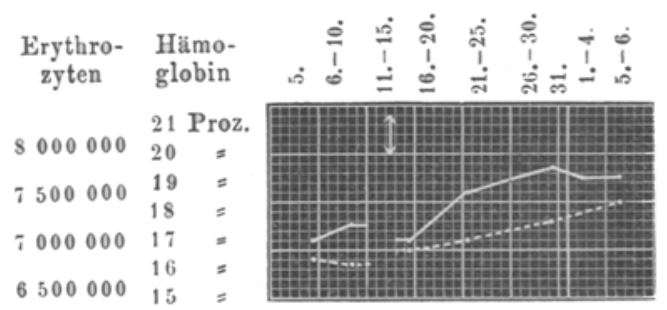

Die Größe der Laparotomiewunde, die Dauer der Narkose, der Blutverlust, entsprachen denjenigen bei den kastrierten Tieren.

Diese Versuche hatten nun das sehr merkwïrdige und auffallende Ergebnis, daß Hämogiobingehalt und Blutkör- 
perebenahl nach dieser Operation nieht nur nicht absanken, sondern im Gegenteil anstiegen, um nach einiger Zeit wieder zu den früheren Werten zurückzukehren. Als Beispiel diene: Hün din Nr. XV.

$\begin{array}{ccc}\text { Datum } & \begin{array}{c}\text { Pote } \\ \text { Blutkörperchen }\end{array} & \begin{array}{c}\text { Absoluter } \\ \text { Hämoglobingehalt }\end{array} \\ \text { 18. April } & 6350000 & 151 / 3 \text { Proz. } \\ \text { 22. Mai } & 6350000 & 15 \quad=\end{array}$

Exstirpation des Uterus am 22. Mai.

$\begin{array}{cllc}\text { 26. Mai } & 6550000 & 16 \text { Proz. } \\ \text { 28. } & 6750000 & 16= \\ \text { 31. } & 7150000 & 17= \\ \text { 4. Juni } & 6750000 & 171 / 2= \\ 9 .= & 7300000 & 172 / 3= \\ 12 .= & 6600000 & 171 / 3= \\ 17 .= & 6300000 & 16=\end{array}$

In diesem, sowie im Fall XIV beträgt die Steigerung des absoluten Hämoglobingehaltes ca. 3 Proz. (=ca. 20 Proz. Fleis chl), die Vermehrung der Körperchenzahl 1-11/2 Millionen. - In Fall XIII wurde zuerst, mit demselben Effekt, die Amputation des Uterus vorgenommen und später die Kastration ausgeführt, die das gewöhnliche Absinken der Blutwerte zur Folge hatte. (Vergl. die Kurve 13, S. 187).

Diese Versuche sind zu wenig zahlreich, als daß wir aus ihnen folgern könnten, die Entfernung des Uterus ftuhre regelmäßig zu einer Steigerung der Blutwerte und bilde also in dieser Beziehung einen der Kastration in ihren Wirkungen entgegengesetzten Eingriff. Aus demselben Grunde möehten wir uns auch aller Deutungsversuche für diese merkwürdige Erseheinung enthalten.

Als Kontrollexperimente zu den Kastrationsversuchen sind die Fälle VIII-XV, sowie Fall XVI (vergl. Kurve 16), in dem in Narkose per laparotomiam ein Stück Netz entfernt wurde, jedesfalls beweisend genug. Sie zeigen, daß das Absinken der Blutwerte bei den kastrierten Tieren kein Effekt des operativen Eingriffs oder der Narkose, sondern zweifellos eine Folge der Entfernung der Ovarien war.

Wir sind uns sehr wohl bewußt, daß die Ergebnisse, über die wir im vorstehenden referiert haben, zu einer Fortsetzung und Variation der Experimente nach verschiedenen Richtungen herausfordern. Es wäre die Abhängigkeit der Wirksamkeit der Kastration vom Alter, resp. der Geschlechtsreife der Tiere genaner zu stu- 
dieren, als wir es bisher getan haben. Es wäre vor allem in Analogie mit den Erfahrungen an Drüsen mit innerer Sekretion (Thyreoidea, Hypophysis usw.) durch das Experiment exakt zu entscheiden, ob die Einverleibung von Orarialsubstanz etwa den entgegengesetzten Einfluß auf die Blutbeschaffenheit äußere wie die Kastration, insbesondere ob das Absinken der Blutwerte nach der Entfernung der Ovarien durch gleichzeitige Behandlung mit Ovarialsubstanz verhütet werden und so der Effekt der Kastration kompensiert werden könne.

Wir haben zu Anfang unserer Versuche bei einem weiblichen Tier durch lange Zeit subkutane Injektionen von Ovarialsubstanz vorgenommen und zwar in Gestalt eines Preßsaftes, den die k. k. Hofapotheke in Wien (Direktor Gut) auf unsere Bitte aus frischen Kälberovarien ${ }^{1}$, durch Zerreiben mit Glaspulver und Auspressen dargestellt hatte. Bei diesem Tier erfolgte ohne weitere Sohädigung des Befindens im Verlauf mehrere Monate ein sehr hohes Ansteiges der Blutwerte (Blutkörperchenzahl bis gegen 9 Millionen).

Doch beweist der eine Versuch natürlich nichts und weitere haben wir nicht angestellt, sondern uns auf die Beobachtung der Folgen der Kastration beschränkt.

Denn alle derartigen Versuche sind, wie uns unsere Erfahrungen gezeigt haben, wenn sie exakt und wirklich beweisend ausgeftihrt werden, - so außerordentlich mulhevoll und zeitraubend, daß wir vorläufig von ihnen absehen mußten. Wir wären natürlich sehr erfreut, wenn sie etwa von anderer Seite aufgenommen und durchgeführt würden.

Auch eine Nachprtifung unserer hier mitgeteilten Kastrationsversuche würden wir natürlich mit Freude begrüßen: doch liegt es in der Natur der Sache, daß dabei alle jene peinlic hen Vorsichtsmaßregeln beobachtet werden mäßten, die wir selbst befolgt haben.

Erst nachdem wir unsere Untersuchungen schon abgeschlossen hatten, gelangten wir zur Kenntnis einiger Abhandlungen, die über ähnliche Versuche berichten. Wie aus dem folgenden Referat sich ergibt, sind diese Untersuchungsreihen zur Entscheidung der Frage nach dem Einfluß der Kastration auf die Blutbeschaffenheit so wenig geeignet, daß wir sie, so weit sie unseren Versuchen widersprechen, wohl kaum als eine Wider-

1) Wiewohl es zweifelhaft erscheinen mußte, daß das Ovarium eines Kalbes so wirkt wie das eines geschlechtsreifen Tieres, haben wir doch aus äußeren gründen dieses Material verwendet. 
legung derselben, und so weit sie mit ihnen übereinstimmen, kaum als eine Bestätigung auffassen muisssen.

Pinz ani (29) fand in einem Versuch bei einer ausgewachsenen Hündin (cagna adulta) nach der Kastration die Zahl der roten Blutkörperchen und das Hämoglobin vermehrt, die Zahl der weißen Blutkörperchen vermindert. Er machte nur eine Blutuntersuchung und zwar - soweit aus seinen Angaben zu entnehmen ist - fruhestens 26 Tage (Heilungsdauer!) nach der Kastration, vielleicht auch später. Es ergab sich eine Zunahme der Erythrozyten um 917,153, eine Zunahme des Hämoglobins um 38 Proz. Fleischl und eine Abnahme der leukozyten um 1919.

Anläßlich seiner Untersuchungen über den Eiweiß- und Fettstoffwechsel nach der Kastration bestimmte Lü thje (31) in einem Versuche an einer 21/2 Jahre alten Hündin die Zahl der roten und weißen Blutzellen und den Hämoglobingehalt des Blutes. Es wurden 13 Monate nach der Kastration (!) an 5 anfeinander folgenden Tagen Blutuntersuchungen vorgenommen. (Die gefundenen Zahlen der Erythrozyten bewegen sich trotzdem zwischen $51 / 2$ und 7 Millionen!) Es ergaben sich keine nennenswerten Differenzen gegenüber einer nicht kastrierten Hündin(!) Über eine Blutuntersuchung des untersuchten Tieres selbst vor der Kastration wird nichts berichtet.

Die Schlüsse, die Pinzani und Lüthje aus ihren Versuchen ziehen, decken sich, wie man sieht, durchaus nicht mit unseren Resultaten; allerdings entsprechen die Versuchsbedingungen auch nicht annähernd den Erfordernissen, die eine experimentelle Untersuchung des Gegenstandes erheiseht, vor allem ist das Intervall zwischen Kastration und Blutuntersuchung ein viel zu langes.

A. Mo nari (30) beschäftigt sich in einer Monographie der Chlorose eingehend mit der Pathogenese dieser Krankheit. Um den vielfach betonten Zusammenhang der Krankheit mit Verändernngen im Sexualapparat zu prïfen, untersuchte er an 6 weiblichen Kaninchen und an 3 Hündinnen den Einfluß der Kastration auf die Blutbeschaffenheit. Die Tiere erholten sich nach der Operation sehr bald und befanden sich dann in bester Verfassung. Von den Kaninchen blieb aber nur eines dauernd am Leben; 5 starben einige Wochen nach der Operation nuter ,komatösen Erscheinungen" ${ }^{1}$ ). Bis zum Tode zeigte sich anläßlich der (nicht allznhäufigen)

1) Sehr merkwürdig ist das sich nach 2-4 wöchentlichem Wohlbefinden bei den kastrierten Kaninchen entwickelnde und binnen wenigen Tagen zum Tode führende Koma, für das auch der Autor keine Erklärung anzugeben vermag. Eine an 4 anderen kastrierten Kaninchen vorgenommene interne und subkutane Behandlung mit Kalbsovariensubstanz bewirkte neben einer vorübergehenden Temperatursteigerung nur ein flüchtiges Aufleben der gesunkenen Körperkräfte, konnte aber den Tod, der wieder unter komatösen Erscheinungen eintrat, nicht einmal hinausschieben. Da man hier immerhin an die Möglichkeit einer Infektion denken mußte, wurden verschiedene Nährböden mit dem Herzblute und der Peritonealfüssigkeit von 2 kastrierten und im Koma verendeten Kaninchen beschickt: alle Platten blieben vollkommen steril. Bei zweizeitiger Ovariotomie (Intervall 2 Monate) blieben die (2) Tiere munter und gediehen sebr gut, wurden aber auffallend lebhaft, reizbar und rauflustig. 
Blutuntersuchungen bei diesen Tieren keine wesentliche Veränderung des Blutbildes gegenüber den Verhältnissen vor der Entfernung der Ovarien.

Die drei kastrierten Händinnen dagegen erholten sich nach der Ope. ration sehr rasch und blieben dauernd gesund. Die ersten Blutuntersuchungen wurden 10 Tage nach der Kastration vorgenommen und ergaben eine Abnahme der Blutwerte bei der

1. Hündin um 3 Millionen rote Blatkörperchen und 5 Proz. Fleischl

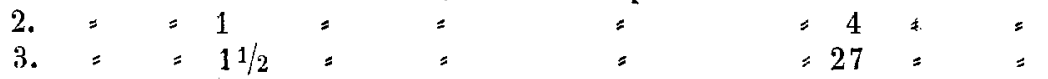

Bei der zweiten Untersuchung (nach weiteren 10 Tagen) waren die Hämoglobinwerte angeblich wieder gleich denen vor der Kastration, während die Körperchenzahl bei Hündin 1 um $1 \frac{1}{2}$, bei Hündin 2 um $1 / 2$ und bei Hündin 3 noch um 1 Million hinter den Zahlen vor der Operation zurïckblieb. Die folgenden Untersuchungen (nach weiteren 14-30 Tagen) ergaben bei allen Tieren wieder normale Werte.

Monaris Resultate stehen also zu den unseren nicht im Gegensatz, denn 10 Tage nach der Kastration ergab sich bei allen 3 Tieren eine recht beträchtliche Abnahme der Erythrozyten und in einem Falle anch des Hämoglobins.

Die Divergenz der beiden Werte bei den Hündinnen 1 und 2 (besonders bei 1) ist allerdings sehr auffallend und läßt die Frage nicht unberechtigt. erscheinen, ob hier nicht irgendwelche Fehler bei den Blutbestimmungen unterlaufen sind, um so mehr, als auch bei der zweiten Blutuntersuchung für das Hämoglobin im Gegensatz zu den Erythrozyten sich normale Werte ergaben. Das rasche Wiederansteigen des Hämoglobins bei der 3. Hündin (nach spätestens 10 Tagen war ein Defizit von 27 Proz. (!) Fleischl wieder ansgeglichen) und das vollkommen normale Verhalten des Blutes in allen 3 Fällen von der 3 . Bestimmung an spricht natürlich durchaus nicht gegen einen Einfuß der Kastration auf die Blutbeschaffenheit; man bedenke nur die großen Intervalle zwischen den einzelnen Blutuntersuchungen (die 3. Blutbestimmung erfolgte bei der ersten Hündin 15, bei der zweiten 13 Tage und bei der dritten 1 Monat [!] nach der 2. Bestimmung) and betrachte einmal z. B. unsere Kurven Nr. I, VII u. XII.

Monari macht über das Alter seiner Versuchstiere keine Angaben; möglicherweise liegt in dem vorgeschrittenen Lebensalter auch eine Ursache für die geringen Wirkungen der Kastrationen bei seinen Hündinnen, vielleicht auch für die vollkommene Unwirksamkeit des Eingriffes in Bezug auf die Blutbeschaffenheit bei seinen Kaninchen. Scheint doch aus unseren Untersuchungen hervorzugehen, da $\beta$ dem Alter der nahenden Geschlechtsreife eine besondere Empfindlichkeit des Blutes gegenüber dem Wegfall der Ovarien zukommt.

Nicht uninteressant namentlich mit Beziehung anf neuere Resultate bei der Kastration sind einige Versuche über die Rondino (32) berichtet. Er injizierte bei weiblichen Kaninchen Glyzerinextrakt von Kuhovarien und fand (allerdings schon bei Blutuntersuchungen wenige Stunden nach der Injektion) eine Vermehrung der Erythrozytenzahl. Dabei soll der Hämoglobingehalt unverändert geblieben sein (?). Bei schwangeren Kaninchen soll die Injektion zu keiner Veränderung im Blutbefund gefülht haben; 
allerdings konnten hier nur geringe Mengen Ovarialsaft injiziert werden, weil dieser anf schwangere Tiere viel giftiger wirkt (eine Angabe, die sich übrigens auch bei Bestion [12] findet). Die Versuche Rondinos sind wohl zu wenig zahlreich und nach ihrer ganzen Anlage zu wenig beweiskräftig, um viel daraus schließen zu können. Immerhin ist der entgegengesetzte Einfluß, den bei ihm die Einverleibung von Ovarialsubstanz gegenüber dem Verlust der Ovarien in unseren Versuchen hervorgerufen hat, nicht ohne Interesse; es stimmen diese Resultate einigermaßen mit dem einen oben erwähnten Versuch, in dem, allerdings bei weit länger fortgesetzter Injektion von Ovarialextrakt, Blutkörperchenzahl and Hämoglobin hoch anstiegen.

Wenn wir uns nun zum Schlusse die Frage vorlegen, was unsere Versuche lehren, so ergibt sich unseres Erachtens folgendes:

Zunächst steht wohl ohne Zweifel fest, daß bei jungen weiblichen Tieren die Kastration einen deutlichen Einfluß auf die Blutbeschaffenheit im Sinne eines Absinkens der Blutwerte ausübt und daß dieses Absinken nicht als eine Folge der operativen Verletzung oder der Narkose, sondern als eine Wirkung des Fortfalls der Ovarien aufzufassen ist. Das übrige Verhalten der kastrierten Tiere macht es außerordentlich wahrscheinlich, daß es sich dabei nicht um eine eigentliche Krankheit, sondern um eine Art von spezifischer isolierter Beeinflussung des Blutes handelt.

Die Blutveränderung ist eine vorübergehende; es macht den Eindruck, als ob das Fehlen der Ovarien seine Wirkung vor allem in der ersten Zeit nach der Kastration zeigte, während nach einiger Zeit etwa dadurch, daß die fragliche Funktion der Eierstöcke von einem anderen Organ übernommen wurde, die alten Blutverhältnisse sich wieder herstellten. Welches dieses vikariierende Organ sein könnte, dafür liegen gar keine Anhaltspunkte vor.

Nicht vollständig und von vorneherein von der Hand weisen läßt sich die Möglichkeit, daß das Absinken der Blutwerte kein reelles sei, sondern nur vorgetäuscht werde etwa durch eine Veränderung in der Blutverteilung (Verarmung des peripheren Kapillarblutes an Körperchen durch vasomotorische Einflüsse etwa im Sinne von Murri (s. o.). Der Parallelismus im Verlauf der Körperchen- und der Hämoglobinkurve könnte daran denken lassen. Doch ist es uns viel wahrscheinlicher, dab es sich um eine wirkliche Verminderung der Körperchen und damit des Farbstoffes bei den operierten Tieren handelt; dafür spricht vor allem das erwähnte Auftreten von kernhaltigen Erytbrozyten zur Zeit des Tiefstandes der Blutwerte.

$\mathrm{Ob}$ die Körperchenverarmung durch einen vermehrten Blut- 
zerfall oder durch eine verminderte Regeneration bedingt sei, dariber ist aus mehreren Versuchen kein Urteil zu gewinnen.

Fragen wir uns endlich, ob dureh unsere Experimente diejenige Theorie eine Stütze erbalte, die das Auftreten der Chlorose zurückführt auf eine ungenügende innere Sekretion der Ovarien. Jene Theorie harmoniert ja mit unseren Resultaten aufs beste Allerdings haben wir, wie wir hier noch einmal hervorheben wollen bei unseren Versuchstieren gewib keine Chlorosen erzeugt; es fehlte neben allen eigentlich krankhaften Erscheinungen vor allem auch die für die menschlicbe Chlorose charakteristische Divergenz zwischen Körperchenzahl und Hämoglobingehalt. Immerhin aber macht es der konstante Ausfall unserer Experimente bei den in der Pubertät stehenden Hündinnen sehr wahrscheinlich, daß auch bei der menschlichen Chlorose einem veränderten Einfluß der Ovarien auf das Blut zum mindesten als einem Teilstücke in dem pathologischen Mechanismus eine wichtige Rolle zufalle.

\section{Literatur.}

1) Rokitansky, Handbuch der speziellen pathologischen Anatomie 1844. Bd. I. S. 418 und 586 .

2) Virchow, Über die Chlorose und die damit zusammenhängenden Anomalien im Gefäßapparate, insbesondere über Endocarditis puerperalis. Beiträge zur Geburtshilfe und Gynäkologie. 1870. Bd. I.

3) E. Fränkel, Über die Kombination von Chlorose mit Aplaxie der weiblichen Genitalorgane. Archiv für Gynäkologie. 1875. Bd. VII.

4) Stieda, Chlorese und Entwicklungsstörungen. Zeitschrift für Geburtshilfe und Gynäkologie. 1895. Bd. XXXII.

5) Beneke, Grundlinien der Pathologie des Stoffwechsels. 1874.

6) Murri, Pathogénie de la chlorose. Semaine medical. 1894. p. 162.

7) Caratulo-Tarulii; Presse médic. 1896. No.66.

8) Senator, Zur Kenntnis der Osteomalacie und Organtherapie. Berliner klin. Wochenschrift. 1897. Nr. 6 u. 7.

9) P. F. Richter, Zur wissenschaftlichen Begrindung der Organtherapie. Deutsche med. Wochenschrift. 1899, Vereinsbeilage Nr. 44. S. 269.

10) Thumim, Über den Eintluß des Oophorius auf den Eiweißumsatz des Menschen. Die Therapie der Gegenwart. 1900. S. 451 .

11) Dałc hé-Lépinois, Bull. médic. 1901. No. 100.

12) Bestion, Presse médic. 1897. p. 203.

13) Neumann und Vas, Über den Einfluß der Ovariumpräparate auf den Stoffwechsel. Monatshefte für Gynäkologie und Geburtshilfe. 1902. Bd. XV.

14) Löw y und Richter, Zur wissenschaftlichen Begründung der Organtherapie. Berliner klin. Wochenschrift. 1899. Nr. 50. Archiv f. Anatomie und Physiologie. 1899. Physiolog. Abteil. Supplem.

15) E. Knauer, Die Ovarientransplantation. Archiv für Gynäkologie. 1900 Bd. LX. H. 2. 
16) Charrin, La chlorose. Gazette hebdomadaire. 1896. No. 1.

17) Leclerc et Levet, Des parentés morbides et de la toxicité du serum dans la chlorose. Lyon médical. 1901. No. 31.

18. Spillmann et Etienne, Sur le traitement de la chlorose par l'ovaréine et le suc ovarien. Sem. méd. 1896. p. 337.

19) Fedeli, Ricerche sull' azione terapeutica dell' ovarina in rapporto ad una nuova teoria della clorosi. Riforma medica, 1896. Octob. p. 218 u. 232.

20) M uret, Organotherapie par l'ovaire. Revue méd. de la Suisse Romande. 1896. No. 7.

21) Blondel, Essai d'une théorie nouvelle de la chlorose. Emploi therapeutique du thymus dans cette affection. Bull. de Thérapie. 1897 23. avril.

22) Salmon, Contributi alla patogenesi della clorosi. Settimana medica. 1897. LI, $20,21$.

23) v. Noorden, Die Bleichsucht. Nothnagels Handbuch der spez. Pathologie und Therapie. VIII, 2. 1897.

24) Etien ne et Demange, La chlorose, antointoxication d'origine ovarielle. Sem. mẻd. 1898. p. 186.

25) Touvenaint, Organothérapie par l'ovaire. Revue internat. de méd. et de chir. 1896. p. 361 .

26) de Gottal, L'opothérapie ovarienne dans la chlorose, les troubles de la menstruation et de la mènopause. Journ. méd. de Bruxelles. 1899. No. 16.

27) Schaumann und v. Willebrand, Einige Bemerkungen über die Blutregeneration bei Chlorose. Berliner klin. Wochenschr. 1899. S. 9 u. 60.

28) Jacobs, Eierstockstherapie. Centralblatt für Gynäkologie. 1897. Nr. 21.

29) Pinzani, Ricerche sperimentali intorno ad alcune modificazioni portate della castrazione ovarica. Archivio ital. di Ginecologia. Napoli 1898. p. 571.

30) Monari, La clorosi. Studio clinico-anatomico. Modena 1900.

31) Lüthje, Über die Kastration und ihre Folgen. Archiv f. experim. Pathol. und Pharmakol. 1902. Bd. XLVIII.

32) Rondino, Azione del succo ovarico sulla crasi sanguigna. Giorn, dell' associazione dei medici naturalisti. Napoli 1901. Anno XI, No. 3. - Jahresbericht für die Fortschritte auf dem Gebiete der Geburtshilfe und Gynäkologie. 1902. 15. Jahrg. S. 266.

33) W. Brünnings, Ein neuer Apparat für Blutkörperchenzählung. Pflügers Archiv 1903. Bd. XCIII. S. 377.

34) E. Veillon, Der Fleischl-Mieschersche Hämometer und die Prifung seiner Leistungsfăhigkeit. - Histochemische und physiologische Arbeiten von F. Miescher. Leipzig, bei F. C. W. Vogel, 1897. Bd. II. S. 423. 